\title{
CMTM5 inhibits the development of prostate cancer via the EGFR/PI3K/AKT signaling pathway
}

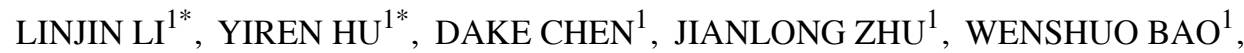 \\ XIAOMIN XU ${ }^{1}, \mathrm{HEYI} \mathrm{CHEN}^{1}, \mathrm{WU} \mathrm{CHEN}^{1}$ and RUI FENG ${ }^{2}$

\begin{abstract}
${ }^{1}$ Department of Urology, The Third Clinical Institute Affiliated to Wenzhou Medical University, The Third Affiliated Hospital of Shanghai University, Wenzhou People's Hospital, Wenzhou, Zhejiang 325000; ${ }^{2}$ Department of Urology, Zhenjiang Hospital of Chinese Traditional and Western Medicine, Zhenjiang, Jiangsu 212002, P.R. China
\end{abstract}

Received January 28, 2021; Accepted July 21, 2021

DOI: $10.3892 / \mathrm{mmr} .2021 .12533$

\begin{abstract}
Prostate cancer (PCa) endangers the life and health of older men. Most PCa cases develop into castration-resistant PCa (CRPC) within 2 years. At present, the molecular mechanisms of the occurrence and development of PCa and its transformation to CRPC remain unknown. The present study aimed to investigate the role of CKLF-like Marvel transmembrane domain containing family member 5 (CMTM5) in PCa and its molecular mechanism in vitro. PCa tissues and paired adjacent normal prostate tissues from 70 patients were collected to examine the expression levels of CMTM5 and EGFR via immunohistochemistry, reverse transcription-quantitative PCR and western blotting. Then, CMTM5-overexpressing DU145 cells were constructed, and CMTM5 expression in these transfected cells and vector control cells was examined via western blotting. Cell Counting Kit- 8 and plate clone formation assays were used to evaluate the proliferation and colony number of CMTM5-overexpressing cells and vector control cells. Then, cell migration and invasion were assessed using wound healing assay, Transwell assay and immunofluorescence analysis with DAPI staining. The effect of CMTM5 on apoptosis and its underlying molecular mechanism were examined using western blotting and flow cytometry. The results demonstrated that CMTM5 expression in PCa tissues and cell lines was significantly downregulated, while EFGR expression was significantly upregulated. The proportion of high CMTM5 expression in PCa tissues was significantly lower compared with that in normal prostate
\end{abstract}

Correspondence to: Dr Rui Feng, Department of Urology, Zhenjiang Hospital of Chinese Traditional and Western Medicine, 18 Tuanshan Road, Zhenjiang, Jiangsu 212002, P.R. China

E-mail: 13913439473@139.com

${ }^{*}$ Contributed equally

Key words: prostate cancer, CKLF-like Marvel transmembrane domain containing family member 5, EGFR, epithelial-mesenchymal transition, PI3K/AKT signaling pathway tissues. By contrast, the proportion of high EGFR expression in PCa tissues was significantly increased compared with that in normal prostate tissues. Moreover, CMTM5 overexpression significantly inhibited cell proliferation, migration and invasion, and promoted cell apoptosis compared with vector control cells in vitro. Furthermore, the regulation of PCa by CMTM5 was associated with the downregulation of $\mathrm{PI} 3 \mathrm{~K} / \mathrm{AKT}$ and its downstream Bcl-2 expression, as well as the upregulation of Bax expression. In conclusion, CMTM5 may be an effective tumor suppressor gene for PCa, especially for castration-resistant $\mathrm{PCa}$, by downregulating EGFR and PI3K/AKT signaling pathway components.

\section{Introduction}

Prostate cancer (PCa) endangers the life and health of older men. The latest statistics on cancer in the United States revealed that the occurrence of PCa was the highest among men, accounting for $>1 / 5$ of newly diagnosed cancer cases (1). With changes to the economy and environment, the incidence of $\mathrm{PCa}$ in China has been rising significantly $(2,3)$. Despite the satisfactory prognosis of $\mathrm{PCa}$ at early stage, most cases inevitably develop into castration-resistant PCa (CRPC) within 2 years, which is accompanied by a poorer prognosis (4). At present, the molecular mechanisms of the occurrence and development of $\mathrm{PCa}$ and its transformation to CRPC remain unknown.

It has been reported that changes in growth factor receptor-related signaling pathways, especially EGFR, may participate in the complex progression of PCa (5). Several researchers have indicated that EGFR is involved in the transition from hormone-dependent PCa to hormone-independent $\mathrm{PCa}$, and is closely associated with the high recurrence rate and poor prognosis of PCa after castration (5-7). In addition, it has been confirmed that EGF can promote the process of epithelial-mesenchymal transition (EMT) in a variety of tumors via the EGFR pathway (8-11). EMT is a complex and vital process of cell biology, which serves an important role in tumor development (10-12). However, the therapeutic effect of EGFR-targeting therapy on patients with CRPC is not satisfactory, indicating that the regulatory mechanism of EGFR in CRPC may be more complex and is worthy of further study $(13,14)$. 
CKLF-like Marvel transmembrane domain containing family member 5 (CMTM5), a member of CMTM, was first reported by the Human Disease Gene Research Center of Beijing University in 2003, and has been shown to significantly inhibit tumor cell proliferation, adhesion and migration $(15,16)$. Other members of the CMTM family, including CMTM3, CMTM7 and CMTM8, have been revealed to inhibit tumor cell proliferation or migration by regulating EGFR signaling pathways (9,17-19). However, it remains unknown whether CMTM5 has a similar regulatory effect in hormone-independent PCa cells, such as CRPC. Therefore, the present study aimed to investigate the role and molecular mechanism of CMTM5 in the development of PCa.

\section{Materials and methods}

Patients and tissue samples. Tissue samples were collected from 70 patients at the Wenzhou People's Hospital between July 2018 and July 2019. All patients were male and the mean age was $61.52 \pm 8.31$ (range, 51-82) years. The distance between the healthy and the cancer tissue was $<5 \mathrm{~cm}$. The inclusion criteria were as follows: Patients with pathologically confirmed primary PCa with different metastasis. The exclusion criteria were as follows: Recurrent PCa, treatment with androgen-deprivation, chemotherapy or radiotherapy prior to sampling, other infectious disease, malignant tumor, severe liver and kidney disease, pulmonary fibrosis, bone metabolic disease, secondary renal hypertension, systemic immune disease or malignant tumor complications. Adjacent tissues in the same patients were also collected as the normal control. The Sixth Edition of the TNM Classification of Malignant Tumors (Union for International Cancer Control) (20) was used for TNM staging. This study was approved by the Ethics Committee of the Wenzhou People's Hospital [Ethics Review (2018) No. (11)], and oral and written informed consent was obtained from all patients according to the committee's regulations. All tissue samples and data were collected and shared in accordance with good medical practices and local laws. The clinical characteristics of all patients are shown in Table I.

Cell culture and transfection. Full-length CMTM5 cDNA (Sino Biological, Inc.) was used to construct the overexpressing CMTM5 lentivirus vector, which was packed using $1.5 \mu \mathrm{g}$ CMTM5 pLVX-IRES-ZsGreen1 plasmid (YouBio), according to manufacturer's protocol (pMDL:VSV-G:REV=5:3:2, multiplicity of infection=20). A 3rd generation system and 293 cell line (Shenzhen Haodi Huatuo Biotechnology Co., Ltd.) were used. The PCa cell line, DU145 (Shenzhen Haodi Huatuo Biotechnology Co., Ltd.), was transfected with the overexpressing CMTM5 lentiviral vector (CMTM5-overexpressing cells) and empty vector (vector control cells) to construct stable transfection. The duration of transduction into cells of interest was 12 h. A flow cytometer (cat. no. 175487; Beckman Coulter, Inc.) was used to detect the expression of CMTM5. Cells were then cultured in the mixture of RPMI-1640 medium (HyClone; Cytiva) containing 10\% FBS (Invitrogen; Thermo Fisher Scientific, Inc.), penicillin (100 U/ml) and streptomycin $(100 \mathrm{U} / \mathrm{ml})$ at $37^{\circ} \mathrm{C}$ with $5 \% \mathrm{CO}_{2}$. Subsequent experiments were performed at $24 \mathrm{~h}$ post-transfection.
Immunohistochemical analysis of tissue samples. Immunohistochemical analysis was performed to detect the cellular localization and expression of CMTM5 and EGFR. Tissue samples were fixed in $4 \%$ paraformaldehyde for $24 \mathrm{~h}$ at room temperature, embedded in paraffin, sectioned to $4-5 \mu \mathrm{m}$ thickness and analyzed via immunohistochemistry. Samples were deparaffinized with xylene, dehydrated with alcohol and subjected to antigen retrieval by boiling samples in decreasing concentrations of EDTA buffer ( $\mathrm{pH} \mathrm{9.0)}$ at $120^{\circ} \mathrm{C}$ for $2 \mathrm{~min}$, followed by cooling to room temperature for $30 \mathrm{~min}$. Then, the sections were soaked in $3 \%$ peroxide solution for $15 \mathrm{~min}$ to quench endogenous peroxidase. After washing in PBS buffer, the sections were blocked with $10 \%$ normal goat serum (Sigma-Aldrich; Merck KGaA) for $10 \mathrm{~min}(\mathrm{pH} 7.5$ ) at room temperature and incubated with rabbit polyclonal to human CMTM5 antibody (1:100; cat. no. ab187980; Abcam) and primary antibodies against EGFR (1:50; cat. no. MA5-13070; Thermo Fisher Scientific, Inc.) at $4^{\circ} \mathrm{C}$ overnight. On the following day, the sections were washed with PBS for $5 \mathrm{~min}$ and incubated with biotin-labeled goat anti-rabbit $\mathrm{IgG}$ (cat. no. ab205718; 1:5,000; Abcam) at $37^{\circ} \mathrm{C}$ for $30 \mathrm{~min}$. The slides were washed and visualized with substrate diaminobenzidine tetrahydrochloride, and then counterstained with hematoxylin at $37^{\circ} \mathrm{C}$ for $1 \mathrm{~min}$. The stained samples were imaged under a light microscope (x20, x50 and x100 magnification; Olympus CX21; Olympus Corporation). According to the percentage of positive cells $(0,<10 \% ; 1,10-24 \%$; $2,25-50 \%$; and $3,>50 \%$ ), the grades of staining intensity were 0 (no staining), 1 (weak staining), 2 (moderate staining) and 3 (strong staining). The CMTM5 score was calculated by the percentage of positive cells $x$ staining intensity, and the range was $0-9$. For all cases, a total score of $\geq 4$ was high expression, a score of 0-3 was low expression.

Reverse transcription-quantitative $(R T-q) P C R$. Total RNA was extracted using TRIzol ${ }^{\circledR}$ reagent (Thermo Fisher Scientific, Inc.) from tissue samples. RNA purity was measured via ultraviolet spectrophotometry, and RNA samples were stored at $-80^{\circ} \mathrm{C}$ for later use. Subsequently, cDNA was synthesized from $1 \mu \mathrm{g}$ RNA using a PrimeScript RT Master Mix kit (Takara Bio, Inc.) in accordance with manufacturer's protocols. The mRNA expression levels of CMTM5, EGFR, AKT, PI3K, Bcl-2 and Bax were quantitatively analyzed using SYBR Green qRT PCR Master mix (Thermo Fisher Scientific, Inc.) on ABI 7300 Plus real-time PCR system (Thermo Fisher Scientific, Inc.). Thermocycling conditions were as follows: Initial hold at $95^{\circ} \mathrm{C}$ for $10 \mathrm{~min}$, followed by 40 cycles of $95^{\circ} \mathrm{C}$ for $15 \mathrm{sec}$ and $60^{\circ} \mathrm{C}$ for $60 \mathrm{sec}$. The mRNA expression level was normalized to $\beta$-actin expression in the same sample. The primers were as follows: CMTM5 forward, 5'-CTTCСТCACСТCCCACAAG-3' and reverse, 5'-AGA TGGAAACCAGGATGATG-3'; EGFR forward, 5'-TTGCCG CAAAGTGTGTAACG-3' and reverse, 5'-GTCACCCCT AAATGCCACCG-3'; AKT forward, 5'-AGCGACGTGGCT ATTGTGAAG-3' and reverse, 5'-GCCATCATTCTTGAG GAGGAAGT-3'; PI3K forward, 5'-TATTTGGACTTTGCG ACAAGACT-3' and reverse, 5'-TCGAACGTACTGGTCTGG ATAG-3'; Bcl-2 forward, 5'-GGTGGGGTCATGTGTGTG G-3' and reverse, 5'-CGGTTCAGGTACTCAGTCATCC-3'; Bax forward, 5'-CCCGAGAGGTCTTTTTCCGAG-3' and 
Table I. Association between CMTM5 expression and clinicopathological features of patients with prostate cancer.

\begin{tabular}{|c|c|c|c|c|c|}
\hline \multirow[b]{2}{*}{ Variables } & \multirow[b]{2}{*}{ Cases $(n=70)$} & \multicolumn{2}{|c|}{ CMTM5 expression } & \multirow[b]{2}{*}{$\chi^{2}$} & \multirow[b]{2}{*}{ P-value } \\
\hline & & High $(n=16)(\%)$ & Low $(\mathrm{n}=54)(\%)$ & & \\
\hline Age, years & & & & 0.102 & 0.750 \\
\hline$<70$ & 43 & $11(25.6)$ & $32(74.4)$ & & \\
\hline$\geq 70$ & 27 & $6(22.2)$ & $21(77.8)$ & & \\
\hline PSA, ng/ml & & & & 0.324 & 0.850 \\
\hline$<4$ & 13 & $9(69.2)$ & $4(30.8)$ & & \\
\hline $4-10$ & 8 & $6(75.0)$ & $2(25.0)$ & & \\
\hline$>10$ & 49 & $32(65.3)$ & $17(34.7)$ & & \\
\hline AFP, ng/ml & & & & 0.251 & 0.616 \\
\hline$<20$ & 32 & $6(18.38)$ & $26(81.2)$ & & \\
\hline$\geq 20$ & 38 & $9(23.7)$ & $29(76.3)$ & & \\
\hline Gleason score & & & & 3.424 & 0.064 \\
\hline$<7$ & 46 & $22(47.8)$ & $24(52.2)$ & & \\
\hline$\geq 7$ & 24 & $6(25.0)$ & $18(75.0)$ & & \\
\hline Status of primary tumor & & & & 0.015 & 0.901 \\
\hline $\mathrm{T} 1-\mathrm{T} 2$ & 21 & $5(23.8)$ & $16(76.2)$ & & \\
\hline T3-T4 & 49 & $11(22.4)$ & $38(77.6)$ & & \\
\hline Clinical stage & & & & 0.004 & 0.947 \\
\hline II & 33 & $11(33.3)$ & $22(66.7)$ & & \\
\hline III-IV & 47 & $16(34.0)$ & $31(66.0)$ & & \\
\hline Lymph node metastasis & & & & 0.102 & 0.749 \\
\hline+ & 28 & $9(32.1)$ & $19(67.9)$ & & \\
\hline- & 42 & $12(28.6)$ & $30(71.4)$ & & \\
\hline Distant metastasis & & & & 0.188 & 0.665 \\
\hline+ & 19 & $7(36.8)$ & $12(63.2)$ & & \\
\hline- & 51 & $16(36.8)$ & $35(31.4)$ & & \\
\hline
\end{tabular}

PSA, prostate-specific antigen; AFP, $\alpha$-fetoprotein; CMTM5, CKLF-like Marvel transmembrane domain containing family member 5.

reverse, 5'-CCAGCCCATGATGGTTCTGAT-3'; and $\beta$-actin (as an internal control) forward, 5'-GGCACTCTTCCAGCC TTCC-3' and reverse, 5'-GAGCCGCCGATCCACAC-3'. The $2^{-\triangle \Delta C q}$ method was used to calculate relative expression of CLCA2 mRNA (21).

Western blotting assay. Total protein was extracted from the tissue samples using RIPA buffer (cat. no. BYL40825; JRDUN Biotechnology) containing protease inhibitor and phosphatase inhibitors, followed by centrifugation at $16,000 \mathrm{~g}$ for $30 \mathrm{~min}$ at $4^{\circ} \mathrm{C}$ to collect the supernatant. Then, samples were separated via $10 \%$ SDS-PAGE and transferred onto a PVDF membrane, and $40 \mu \mathrm{g}$ protein/lane was separated via $8-12 \%$ SDS-PAGE. The membrane was blocked with $5 \%$ non-fat milk in TBS at $37^{\circ} \mathrm{C}$ for $2 \mathrm{~h}$, and then incubated with antibodies against CMTM5 (1:500; cat. no. ab187980; Abcam), EGFR (1:500; cat. no. MA5-13070; Thermo Fisher Scientific, Inc.), p-EGFR (1:200; cat. no. 44-788G; Thermo Fisher Scientific, Inc.) and $\beta$-actin $(1: 1,000$; cat. no. ab8226; Abcam) at $4^{\circ} \mathrm{C}$ overnight. After three washes in $0.05 \%$ TBS-Tween-20, the membrane was incubated with HRP-conjugated goat anti-rabbit secondary antibody (1:5,000; cat. no. sc2030; Santa Cruz Biotechnology, Inc.) and HRP-conjugated Affinipure Goat Anti-Mouse IgG (1:2,500, cat. no. SA00001-1; ProteinTech Group, Inc.), at room temperature for $1 \mathrm{~h}$. According to the manufacturer's instructions, subsequent detection was performed using the ECL western blotting system (Amersham; Cytiva).

Western blotting was also used to detect the relative protein expression level of CMTM5 in CMTM5-overexpressing cells and vector control cells. The procedure was repeated as aforementioned.

Moreover, primary antibodies against $\beta$-actin $(1: 1,000$; Abcam; cat. no. ab8226), AKT (1:800; cat. no. 4691; Cell Signaling Technology, Inc.), phosphorylated (p)-AKT (1:800; cat. no. 4051; Cell Signaling Technology, Inc.), PI3K (1:800; cat. no. ab86714; Abcam), Bcl-2 (1:200; cat. no. ab117115; Abcam) and Bax (1:200; cat. no. ab32503, Abcam) were used detect cell apoptosis. The procedure was repeated as described previously.

ImageJ software (version 1.46; National Institutes of Health) was used to measure the grey values of protein bands. 
Wound healing assay. A wound healing assay was performed to assess the effect of CMTM5 on cell lateral migration. CMTM5-overexpressing cells and vector control cells were placed in 6-well plates (500 1/well). A scratch was created using a $100 \mu$ l-sterile micropipette tip. Cells were then washed twice with PBS to remove cellular debris and cultured at $37^{\circ} \mathrm{C}$ in serum-free DMEM for $24 \mathrm{~h}$. Cells were imaged using an inverted light microscope (x20 magnification, DM IL; Leica Microsystems $\mathrm{GmbH}$ ) at 0 and $48 \mathrm{~h}$, and the wound width was analyzed using ImageJ software 1.46 (National Institutes of Health).

Transwell migration and invasion assays. The Transwell assay was performed to assess the efficacy of CMTM5 on cell longitudinal migration and invasion. CMTM5-overexpressing cells and vector control cells were placed in common Transwell plate (Corning, Inc.) chambers to evaluate the migratory ability. Cells were resuspended in $200 \mu \mathrm{l}$ serum-free DMEM, and the cell suspension was then added into the upper chambers ( $1 \times 10^{5}$ cells/well) for $24 \mathrm{~h}$, and DMEM containing $10 \% \mathrm{FBS}$ was added to the lower chambers $(600 \mu \mathrm{l} /$ well). After culturing at $37^{\circ} \mathrm{C}$ in $5 \% \mathrm{CO}_{2}$ for $48 \mathrm{~h}$, the membrane was washed with PBS, fixed in $4 \%$ paraformaldehyde at room temperature for $30 \mathrm{~min}$ and stained with $0.1 \%$ crystal violet at room temperature for $10 \mathrm{~min}$. Cells that did not pass through the polycarbonate membrane were scraped away gently with a cotton swab, and the number of migrated cells was counted using an inverted light microscope (MSHOT/MC30 Digital imaging system; Guangzhou Micro-shot Technology Co., Ltd.) under a x200 magnification.

The invasive ability of cells was determined in the same approach, but the cells were added into upper chambers precoated with Matrigel (MilliporeSigma) at room temperature for $15 \mathrm{~min}$.

Cell Counting Kit-8 (CCK-8) assay. CMTM5-overexpressing cells and vector control cells were inoculated in 96 -well plates in triplicate at the density of $2 \times 10^{3}$ cells/well, and cultured at $37^{\circ} \mathrm{C}$. Then, $10 \mu \mathrm{lCCK}-8$ solution (Tomos Biotools Shanghai Co., Ltd.) was added to each well at the indicated time points according to the manufacturer's instructions following transfection for $2 \mathrm{~h}$ at $37^{\circ} \mathrm{C}$. Cells were incubated for $2 \mathrm{~h}$ in a $5 \% \mathrm{CO}_{2}$ incubator at $37^{\circ} \mathrm{C}$. The number of viable cells was calculated via absorbance measurements at $570 \mathrm{~nm}$ using a microplate reader (Bio-Rad Laboratories, Inc.).

Plate clone formation assay. CMTM5-overexpressing cells and vector control cells in the logarithmic phase were selected and digested with $0.05 \%$ trypsin into single cells. Cells were suspended in RPMI-1640 medium containing 10\% FBS, and the cell concentration was adjusted to $1 \times 10^{3}$ cells $/ \mathrm{ml}$. In total, $200 \mu \mathrm{l}$ CMTM5-overexpressing cell and vector control cell suspensions were seeded into culture dishes $(60-\mathrm{mm} ; 200$ cells per dish) and complete medium was added to $10 \mathrm{ml}$. The plates were incubated in a cell incubator with $5 \% \mathrm{CO}_{2}$ at $37^{\circ} \mathrm{C}$ for 2-3 weeks. Then, cells were fixed in $5 \mathrm{ml}$ methanol for $15 \mathrm{~min}$ at room temperature and dyed with Giemsa stain for $20 \mathrm{~min}$ at room temperature. Then, $0.1 \%$ crystal violet staining solution was used for staining for $30 \mathrm{~min}$ at room temperature, and then the stain was washed away with double distilled water and samples were allowed to dry naturally. A transparent film with grids was placed under a culture dish and the number of colonies (>50 cells) was counted using a light microscope (magnification, x100; Olympus Corporation).

Immunofluorescence. CMTM5-overexpressing cells and vector control cells were subjected grown on glass slides, washed with PBS and fixed in using $4 \%$ buffered paraformaldehyde dissolved in $0.2 \mathrm{M}$ phosphate buffer $(\mathrm{pH} 7.3$ ) for $15 \mathrm{~min}$ at room temperature. The samples were then blocked with $2 \%$ BSA (Sigma-Aldrich; Merck KGaA) for $1 \mathrm{~h}$ at room temperature and incubated overnight with p-EGFR (1:200; cat. no. 44-788G; Thermo Fisher Scientific, Inc.) or E-cadherin primary antibodies (1:200; cat. no. 14-3249-82; Thermo Fisher Scientific, Inc.). After washing three times with PBS, the samples were incubated in a dark chamber with fluorochrome-conjugated secondary antibody (1:400; cat. no. 4408S; Cell Signaling Technology, Inc.) for $2 \mathrm{~h}$ at room temperature. The samples were washed with PBS and cover slips were mounted with $90 \%$ glycerol in PBS. DAPI (Roche Diagnostics) was used for nuclear staining for $10 \mathrm{~min}$ at room temperature. The fluorescent images were observed under a fluorescence microscope (magnification, x100, Nikon TE-2000U; Nikon Corporation). The total area of target marker-positive cells was then evaluated using ImageJ software (version 1.8.0, National Institutes of Health).

FITC-Annexin V/PI double staining. CMTM5-overexpressing cells and vector control cells were digested with trypsin and both were either stimulated with or without the AKT inhibitor MK-2206 (50 nmol/l; MedChem Express) for $2 \mathrm{~h}$ at room temperature. On this basis, the four samples were plated in 96-well plates at a density of $1 \times 10^{4}$ cells/well in triplicate. The apoptosis rate of cells was evaluated via Annexin V FITC/PI staining using corresponding apoptosis detection kits (BD Biosciences). The cells were stained with $5 \mu$ l Annexin V FITC and $5 \mu \mathrm{l}$ PI and incubated at room temperature in the dark for $15 \mathrm{~min}$. The extent of apoptosis was analyzed using a flow cytometer (FACScan; BD Biosciences). Data were analyzed using FlowJo software V10.7.1 (FlowJo, LLC).

Statistical analysis. Each experiment was repeated three times. SPSS 20.0 statistical software (IBM Corp.) and GraphPad Prism software (version 8.0.2; GraphPad Software, Inc.) were used for data analysis. ImageJ software (v1.53a, National Institutes of Health) was used to analyze the image results of cell experiments. All data are presented as the mean \pm SD. The results were accessed using a $\chi^{2}$ test, paired t-test, unpaired t-test and one-way ANOVA followed by Bonferroni correction comparisons. $\mathrm{P}<0.05$ was considered to indicate a statistically significant difference.

\section{Results}

CMTM5 expression is downregulated in PCa tissues. CMTM5 was predominately localized in the cytoplasm (Fig. 1A). The negative expression, weak expression and medium or strong expression of CMTM5 in prostate tumor tissue samples were $47.1 \%$ (33/70), 35.7\% (25/70) and 17.1\% (12/70), respectively. By contrast, CMTM5 in the 70 normal prostate tissue samples was either strongly $(37 / 70 ; 52.9 \%)$ or moderately $(30 / 70 ; 42.9 \%)$ 
A

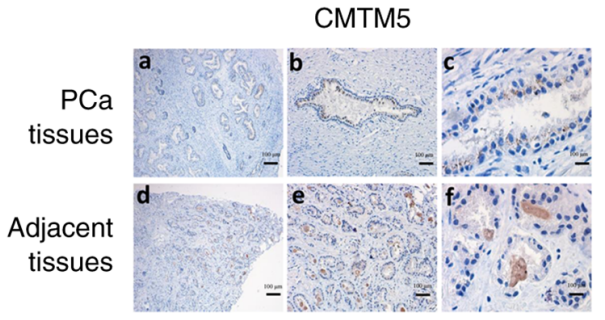

B

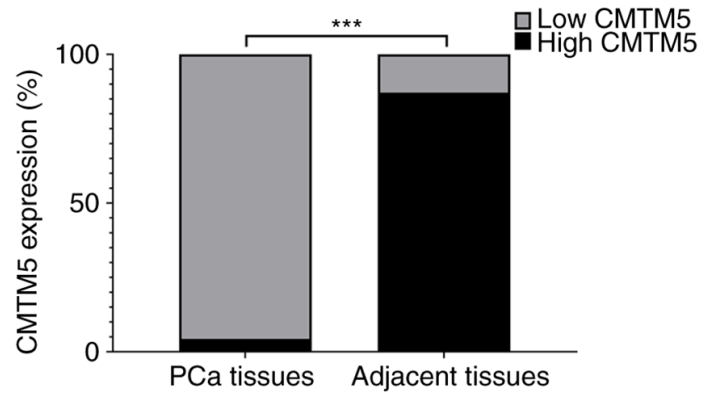

C

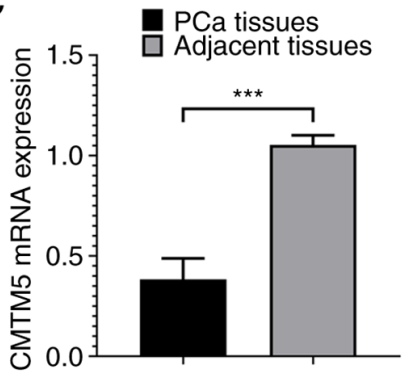

D

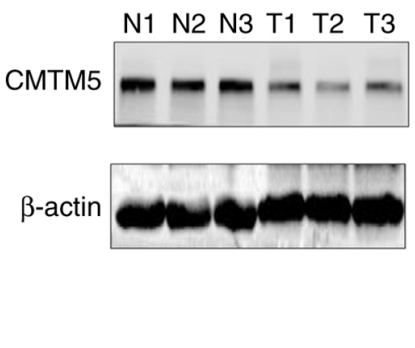

E

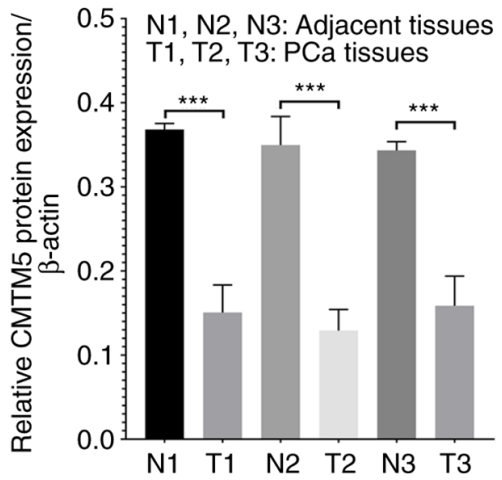

Figure 1. CMTM5 expression is downregulated in PCa tissues. (A) Representative images of CMTM5 staining in PCa and adjacent prostate tissues, (a) PCa tissues in x20, (b) PCa tissues in x40, (c) PCa tissues in x100, (d) adjacent prostate tissues in x20, (e) adjacent prostate tissues in x40, (f) adjacent tissues in x100 magnification. Scale bars, 100, 50 and $20 \mu \mathrm{m}$. (B) The ratio of high CMTM5 expression and low CMTM5 expression between PCa and adjacent prostate tissues (C) CMTM5 mRNA expression in PCa and adjacent prostate tissues was detected via reverse transcription-quantitative PCR. (D) Western blotting results of CMTM5 protein expression in PCa and adjacent prostate tissues. (E) The gray degree values of CMTM5 western blotting results. ${ }^{* * *} \mathrm{P}<0.001$. PCa, prostate cancer; CMTM5, CKLF-like Marvel transmembrane domain containing family member 5; N, normal; T, tumor.

expressed, with only a few with weakly positive staining $(3 / 70$; 4.3\%). This indicated that the high expression of CMTM5 was significantly lower in $\mathrm{PCa}$ tissues compared with that in normal prostate tissues $(\mathrm{P}<0.001$; Fig. 1B). Western blotting and RT-qPCR results suggested that the protein and mRNA expression levels of CMTM5 were significantly downregulated in $\mathrm{PCa}$ tissues compared with adjacent tissues (both $\mathrm{P}<0.001$; Fig. 1C-E).

EGFR expression is upregulated in PCa tissues. The immunohistochemistry results identified that EGFR was mainly localized on the cell biofilm system, such as plasma membrane, endoplasmic reticulum and vacuolar, as well as a small amount on the nuclear and cytoplasmic matrix (Fig. 2A). In the $70 \mathrm{PCa}$ tissues, 64 samples (91.4\%) showed high EGFR expression and only 6 samples $(8.6 \%)$ showed low EGFR expression. However, in the 70 adjacent prostate tissues, low EGFR expression was found in 39 samples (55.7\%) and high EGFR expression in 31 samples $(44.3 \%)(\mathrm{P}<0.01$; Fig. $2 \mathrm{~B})$. The RT-qPCR results demonstrated that EGFR mRNA expression was upregulated in PCa tissues ( $\mathrm{P}<0.001$; Fig. $2 \mathrm{C})$. To further confirm this finding, EGFR and p-EGFR expression was detected using western blotting, and found that the protein expression levels of EGFR and p-EGFR were upregulated in PCa tissues (both $\mathrm{P}<0.05$; Fig. 2D and $\mathrm{E}$ ).

CMTM5 inhibits the proliferation, migration and invasion of PCa cells. To determine the biological function of CMTM5 in pathogenesis of PCa, CMTM5 overexpression was induced in DU145 cells. As shown in Fig. 3A, the lentivirus-mediated overexpression was established, and a higher expression of CMTM5 protein was detected in CMTM5-overexpressing cells $(\mathrm{P}<0.001)$. CCK-8 and clone formation assays indicated that the proliferation and colony number of cells were significantly decreased in CMTM5 overexpression group ( $\mathrm{P}<0.05$ and $\mathrm{P}<0.001$, respectively; Fig. $3 \mathrm{~B}$ and $\mathrm{C})$. Moreover, the wound healing assay revealed that CMTM5-overexpressing cells exhibited a lower scratch closure rate compared with that of vector control cells $(\mathrm{P}<0.001$; Fig. 3D). Similarly, the results of the Transwell migration assay indicated that CMTM5 overexpression significantly reduced the number of migrated cells $(\mathrm{P}<0.001$; Fig. 3E). Furthermore, the Transwell invasion assay was conducted to evaluate the effect of CMTM5 overexpression on the invasive ability of DU145 cells, and it was found that CMTM5 overexpression inhibited cell invasion $(\mathrm{P}<0.001$; Fig. 3E). Taken together, these results suggested that CMTM5 inhibited cell proliferation, migration and invasion in vitro.

CMTM5 overexpression increases E-cadherin and decreases p-EGFR in PCa cells. As detected via immunofluorescence, CMTM5 overexpression led to a significant upregulation of E-cadherin expression when compared with control group (Fig. 4A). On the contrary, p-EGFR was negatively expressed in CMTM5-overexpressing cells, but positively expressed in vector control cells (Fig. 4B). Both the results indicated that CMTM5 may inhibit metastasis of PCa according to the expression of the analyzed marker proteins.

CMTM5 promotes PCa cell apoptosis by downregulating the PI3K/AKT signaling pathway. As shown in Fig. 5A, CMTM5 
A

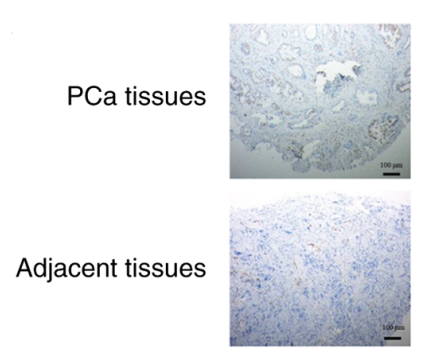

C

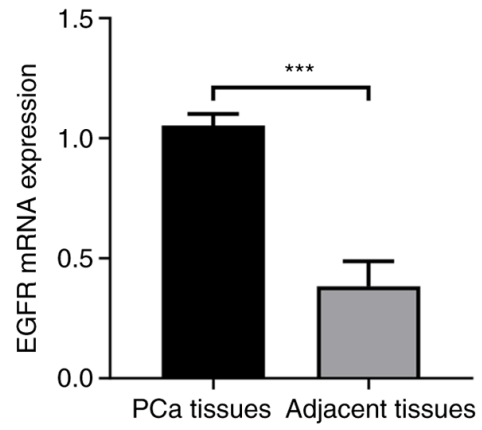

EGFR

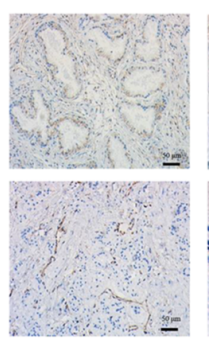

D

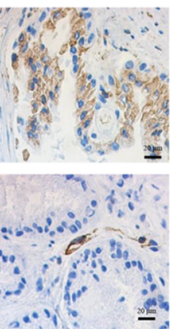

E
$\mathrm{B}$
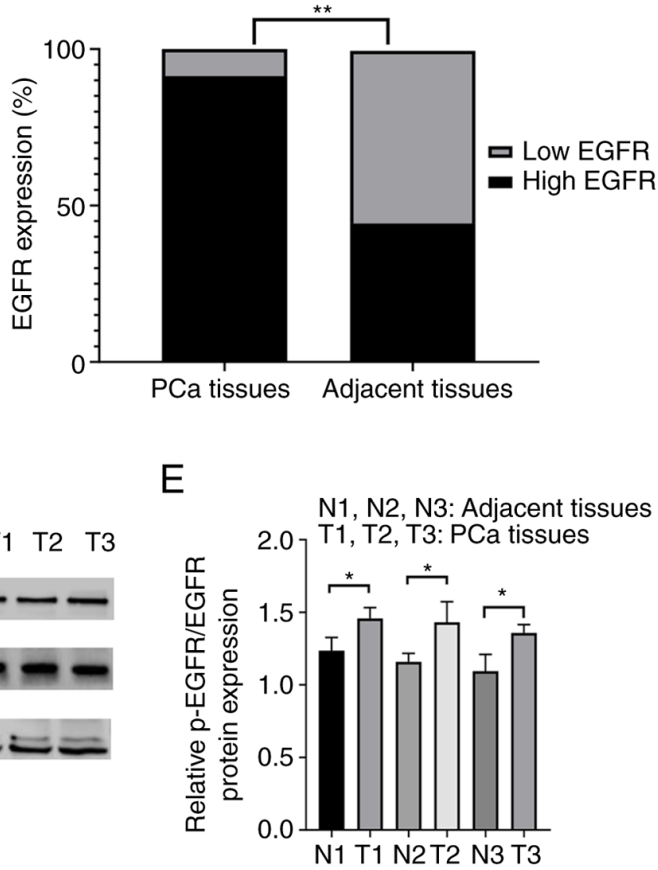

Figure 2. EGFR expression is upregulated in PCa tissues. (A) Representative images of EGFR staining in PCa and adjacent prostate tissues. Scale bars, 100, 50 and $20 \mu \mathrm{m}$. (B) The ratio of high EGFR expression and low EGFR expression between PCa and adjacent prostate tissues. (C) EGFR mRNA expression in PCa and adjacent prostate tissues was assessed via reverse transcription-quantitative PCR. (D) Western blotting results of EGFR and p-EGFR protein expression in PCa and adjacent prostate tissues. (E) The gray degree values of $\mathrm{p}$-EGFR/EGFR western blotting results. ${ }^{*} \mathrm{P}<0.05,{ }^{* * *} \mathrm{P}<0.01,{ }^{* * *} \mathrm{P}<0.001$. PCa, prostate cancer; $\mathrm{N}$, normal; T, tumor; p-, phosphorylated.
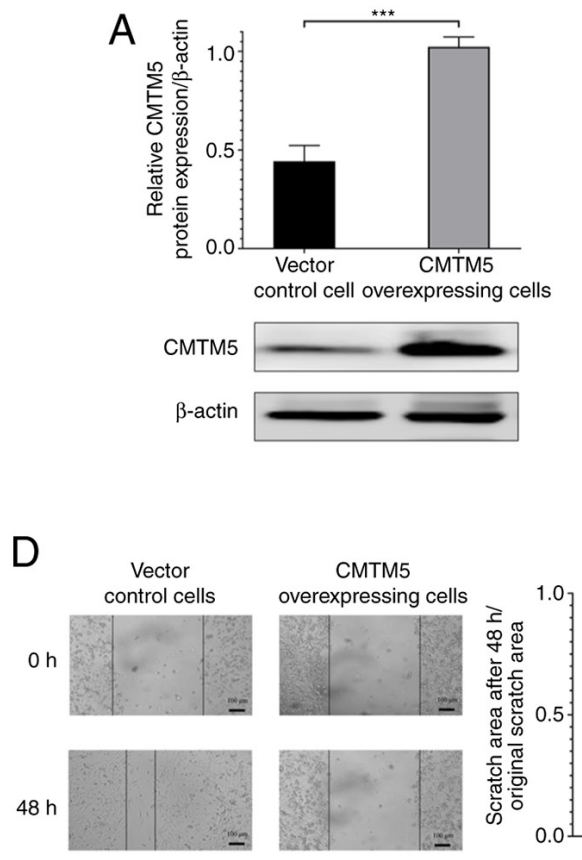
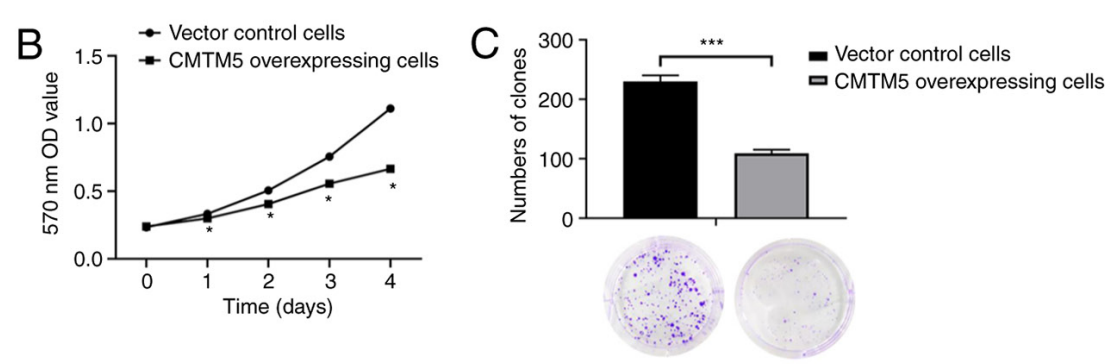

$\mathrm{E}$

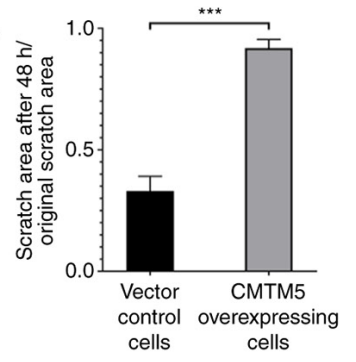

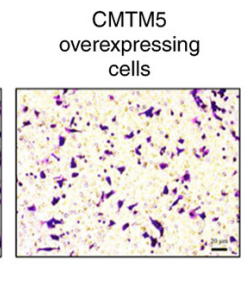
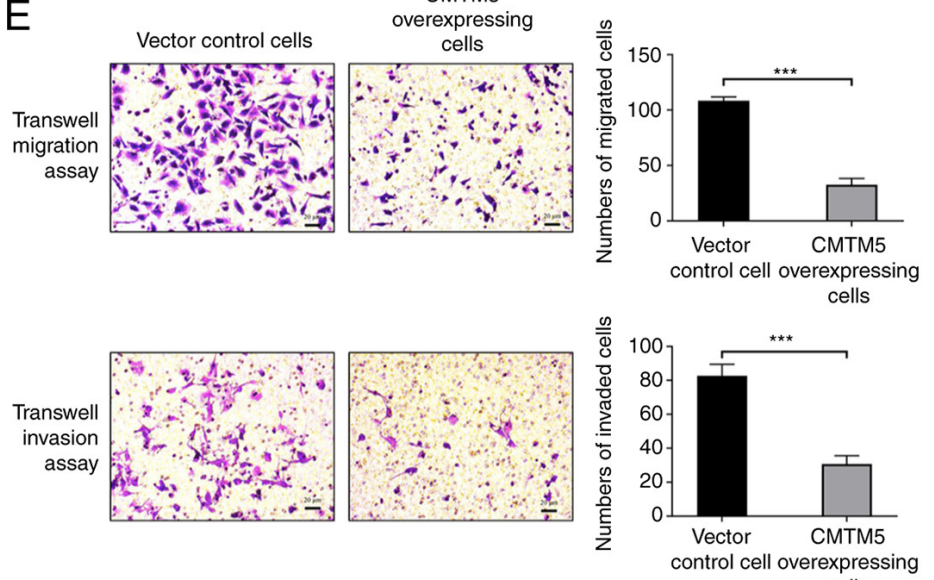

cells

Figure 3. Effect of CMTM5 on DU145 cells. (A) Western blot analysis of relative CMTM5 protein expression in CMTM5-overexpressing cells and vector control cells. ${ }^{* * *} \mathrm{P}<0.001$. (B) Cell Counting Kit-8 assay results of the proliferation of CMTM5-overexpressing cells and vector control cells. ${ }^{*} \mathrm{P}<0.05$ vs vector control cells. (C) Colony-forming capacity was measured by counting number of colonies with $\geq 50$ cells. ${ }^{* * *} \mathrm{P}<0.001$. (D) Wound healing assay of DU145 cells transfected with overexpressing CMTM5 lentivirus vector. ${ }^{* * *} \mathrm{P}<0.001$. Scale bar, $100 \mu \mathrm{m}$. (E) Transwell migration and invasion assays were performed to evaluate the impact of CMTM5 on the migration and invasion of PCa cells. Scale bar, $20 \mu \mathrm{m} .{ }^{* * *} \mathrm{P}<0.001$. Values are expressed as the mean \pm SD of the mean and are representative of three independent experiments. PCa, prostate cancer; CMTM5, CKLF-like Marvel transmembrane domain containing family member 5; OD, optical density. 
A

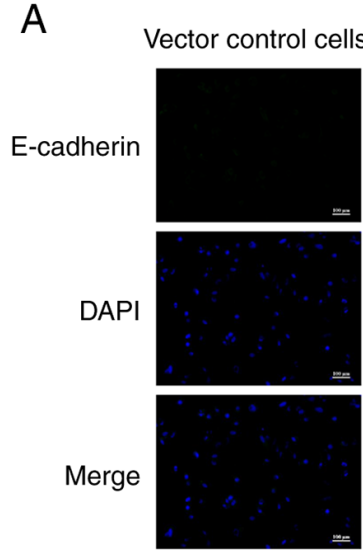

CMTM5 overexpressing cells
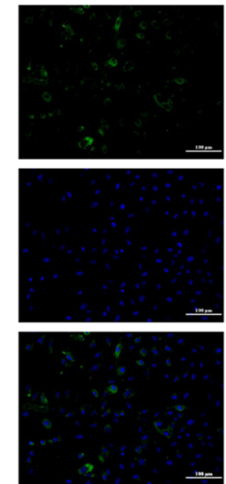

B

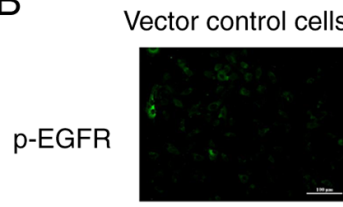

DAPI

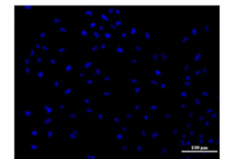

Merge

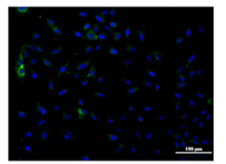

CMTM5 overexpressing cells
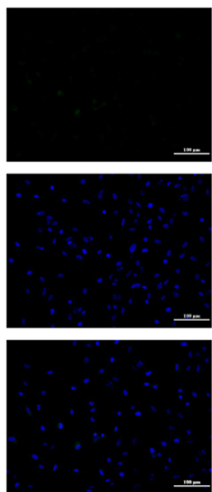
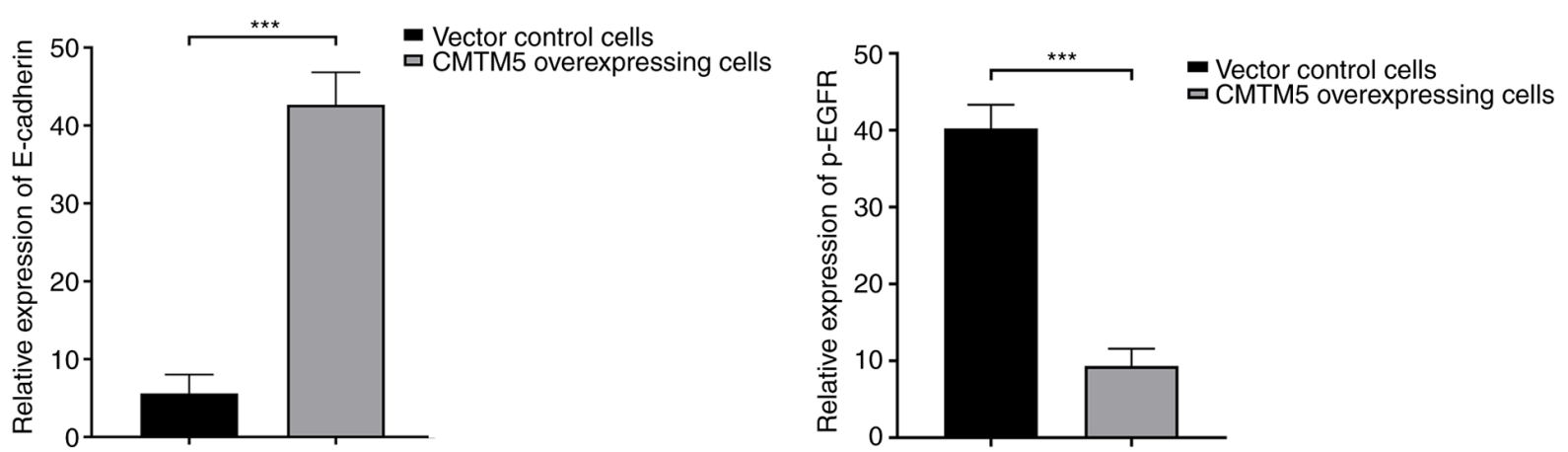

Figure 4. Immunofluorescence analysis with DAPI staining on DU145 cells. (A) Expression of E-cadherin in CMTM5-overexpressing cells and vector control cells. (B) Expression of p-EGFR in CMTM5-overexpressing cells and vector control cells. Scale bar, $100 \mu \mathrm{m}$. ${ }^{* * *} \mathrm{P}<0.001$. CMTM5, CKLF-like Marvel transmembrane domain containing family member 5 ; p-, phosphorylated.

overexpression significantly downregulated the protein expression levels of p-AKT, PI3K and $\mathrm{Bcl}-2$ and upregulated Bax protein expression $(\mathrm{P}<0.001)$ when compared with the control group. Vector control cells treated with the AKT inhibitor MK-2206 had higher early apoptosis, late apoptosis and necrosis rates compared with those without MK-2206 $(\mathrm{P}<0.001$; Fig. 5B). The late apoptosis and necrosis rates of CMTM5-overexpressing cells treated with MK-2206 were significantly higher compared with those without MK-2206 $(\mathrm{P}<0.001$; Fig. 5B). These findings indicated CMTM5 could promote apoptosis by downregulating the PI3K/AKT signaling pathway components in PCa cells.

\section{Discussion}

CMTM5, located at 14q11.2, is a polygenic locus associated with various cancer types. For example, in nasopharyngeal carcinoma, the frequent loss of heterozygosity at 14q11.2 indicates the presence of functional tumor-suppressor gene at this locus, suggesting that CMTM5 may be involved in tumorigenesis (22). The present study aimed to investigate the effect of CMTM5 on tumorigenesis of PCa in vitro.

Previous studies have revealed that the expression level of CMTM5 was reduced or undetectable in most tumor cell lines or tissues $(23,24)$. Similarly, the current study found that CMTM5 expression was significantly downregulated in PCa tissues when compared with normal prostate cancer tissues. Moreover, EGFR expression was upregulated in $\mathrm{PCa}$ tissues. By contrast, p-EGFR was significantly decreased in
CMTM5-overexpressing cells. Other members of the CMTM gene family have been shown to serve a role in human cancer by influencing EGFR and EGFR-related signaling pathways. Yuan et al (9) reported that CMTM3 inhibited EGF-mediated tumorigenicity of gastric cancer cells by reducing EGFR expression and promoting EGFR degradation. In addition, the loss of CMTM7 in non-small cell lung cancer contributes to the maintenance of aberrant EGFR-mediated oncogenic signaling (18), whereas enhanced expression of CMTM7 inhibits the proliferation and migration of hepatoma cells (25). Thus, we hypothesized that CMTM5 may be able to affect the expression of EGFR in PCa.

In the present study, E-cadherin expression was upregulated in CMTM5-transfected DU145 cells, while p-EGFR was negatively expressed. It has been reported that EMT serves an important role in the development and metastasis of PCa (26), and EGFR can mediate the occurrence of EMT in human cancer types, including PCa $(10,27)$. Moreover, the expression changes of related markers, such as E-cadherin, are vital characteristics of EMT (28). Aberrant EMT activation may trigger the dissociation of cancer cells from the primary cancer and migrate to distant organs (29). During this process, the expression of cell adhesion molecules, including E-cadherin, is suppressed (29). Combined with the experimental findings of clinical samples, the current immunofluorescence results demonstrated that CMTM5 downregulated EGFR-related proteins in $\mathrm{PCa}$, thereby inhibiting the occurrence of the EMT response and further suppressing the proliferation and invasion of PCa cells. In combination with previous studies (8-11), 

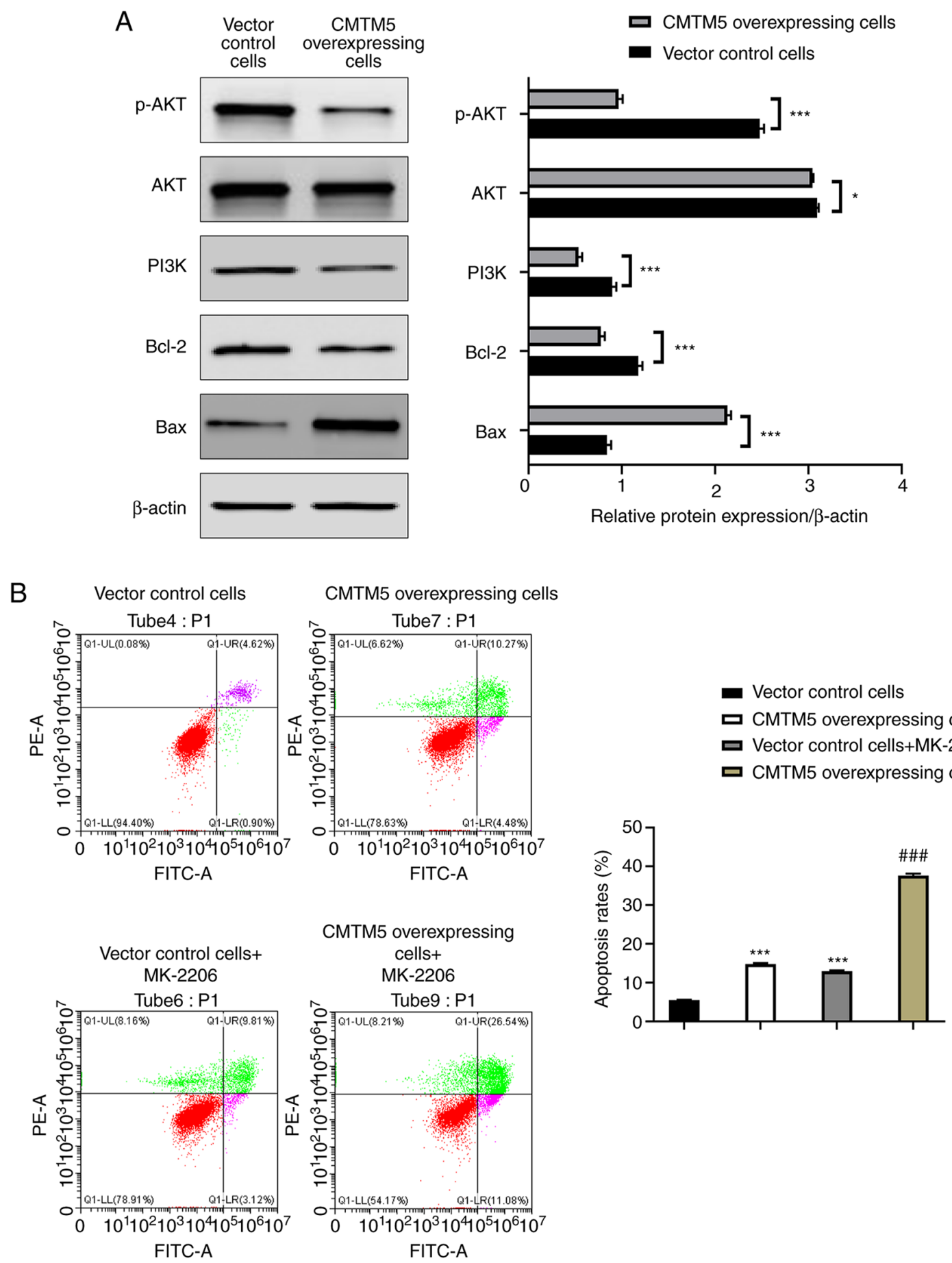

Figure 5. Effect of CMTM5 on apoptosis of DU145 cells. (A) Western blot analysis of p-AKT/AKT, PI3K, Bcl-2 and Bax protein expression in CMTM5-overexpressing cells and vector control cells. Bars represent the mean \pm SD of three independent experiments. ${ }^{* * *} \mathrm{P}<0.001$. (B) The apoptotic rate in each group following transfection was measured via flow cytometry. ${ }^{*} \mathrm{P}<0.05,{ }^{* * * *} \mathrm{P}<0.001$ vs. vector control; ${ }^{\# \# \#} \mathrm{P}<0.001$ vs. CMTM5-overexpressing cells. CMTM5, CKLF-like Marvel transmembrane domain containing family member 5; p-, phosphorylated.

the current study demonstrated that the ability to downregulate EGFR may prevent the conversion of PCa to CRPC, which provided a novel idea for the treatment of this conversion.

A previous study revealed that CMTM3 from the same gene family as CMTM5 can inhibit migration, invasion and proliferation of the PCa cell line LNCaP (30). A study conducted by Guan et al (31) examined the relationship between CMTM5 and the proliferation, migration and invasion of hepatocellular carcinoma cells (HCC), and found that the inhibition of CMTM5 expression by microRNA-10b-3p promoted the progression of HCC cells, whereas CMTM5 overexpression reduced cell proliferation, migration and invasion (31). Consistently, the present findings revealed that the proliferation, migration and invasion of DU145 cells were decreased after transfection with CMTM5, demonstrating that CMTM5 had a regulatory effect on $\mathrm{PCa}$ cell proliferation, migration and invasion. At the same time, PCa cells transfected with the CMTM5 overexpressing vector had a higher apoptotic rate 
compared with cells transfected with empty vectors. Several researches have shown that CMTM5 can induce the apoptosis of renal and pancreatic cancer cells $(23,32)$, which was consistent with the current findings.

It has been reported that the PI3K/AKT signaling pathway is involved in the occurrence and development of PCa, and ectopic expression of CMTM5 in PCa cells can decrease Akt activity $(33,34)$. Phosphorylation of PI3K activates the phosphorylation of Akt, which have central roles in regulating cell proliferation and survival (35). In the present study, the western blotting results suggested that the protein expression levels of p-AKT, AKT and PI3K were downregulated in CMTM5-overexpressing cells. In addition, both CMTM5-overexpressing cells and vector control cells had significantly increased apoptosis, and the apoptosis of CMTM5-overexpressing cells was further enhanced by the addition of the AKT inhibitor MK-2206. This indicated that CMTM5 inhibited PCa progression via the inhibition of the PI3K/AKT signaling pathway.

Compared with other studies, to the best of our knowledge, the present study was the first to examine how CMTM5 exerts its anticancer effect on PCa by inhibiting EMT, providing a new direction for the future clinical treatment of $\mathrm{PCa}$ and prevention of the conversion of PCa to CRPC. However, the current study did not conduct in vivo experiments, and the in vivo results may differ from those in this study. In addition, it remains difficult to analyze the association of both EGFR and CMTM5 between the same individuals. These issues will be addressed in future research, when more in-depth studies will be conducted. Moreover, the correlation between the expression of CMTM5 and the corresponding characteristics of tumors in various PCa cell lines will be investigated, with the aim of elucidating the biologically relevant functions of CMTM5.

In conclusion, the present study provided evidence to demonstrate that CMTM5 expression was downregulated in $\mathrm{PCa}$ and exerts an anticancer role by regulating the EGFR/PI3K/AKT signaling pathways. Thus, CMTM5 may be the starting point of a novel therapeutic strategy for the treatment of $\mathrm{PCa}$.

\section{Acknowledgements}

Not applicable.

\section{Funding}

This work was supported by the Medical Health and Scientific Program of Zhejiang province (grant no. 2019RC277).

\section{Availability of data and materials}

The datasets used and/or analyzed during the present study are available from the corresponding author on reasonable request.

\section{Authors' contributions}

LL, YH and RF conceived the study. DC developed the methodology. JZ performed the experiments. WB, XX, HC and WC analyzed and interpretated data. All authors have read and approved the final manuscript. LL and YH confirm the authenticity of all the raw data.

\section{Ethics approval and consent to participate}

This study was approved by the Ethics Committee of the Wenzhou People's Hospital. Informed consent was obtained from all patients.

\section{Patient consent for publication}

Not applicable.

\section{Competing interests}

The authors declare that they have no competing interests.

\section{References}

1. Siegel RL, Miller K and Jemal A: Cancer statistics, 2020. CA Cancer J Clin 70: 7-30, 2020.

2. Zhuo L, Cheng Y, Pan Y, Zong J, Sun W, Xu L, Soriano-Gabarró M, Song Y, Lu J and Zhan S: Prostate cancer with bone metastasis in Beijing: An observational study of prevalence, hospital visits and treatment costs using data from an administrative claims database. BMJ Open 9: e028214, 2019.

3. Jia Y, Zhu LY, Xian YX, Sun XQ, Gao JG, Zhang XH, Hou SC, Zhang CC and Liu ZX: Detection rate of prostate cancer following biopsy among the northern Han Chinese population: A single-center retrospective study of 1022 cases. World J Surg Oncol 15: 165, 2017.

4. Wu G, Huang S, Nastiuk KL, Li J, Gu J, Wu M, Zhang Q, Lin H and Wu D: Variant allele of HSD3B1 increases progression to castration-resistant prostate cancer. Prostate 75: 777-782, 2015.

5. Mimeault M and Batra SK: Recent advances on multiple tumorigenic cascades involved in prostatic cancer progression and targeting therapies. Carcinogenesis 27: 1-22, 2006.

6. Peraldo-Neia C, Migliardi G, Mello-Grand M, Montemurro F, Segir R, Pignochino Y, Cavalloni G, Torchio B, Mosso L, Chiorino G and Aglietta M: Epidermal Growth Factor Receptor (EGFR) mutation analysis, gene expression profiling and EGFR protein expression in primary prostate cancer. BMC Cancer 11: 31, 2011.

7. Mitsunari K, Miyata Y, Asai A, Matsuo T, Shida Y, Hakariya T and Sakai H: Human antigen R is positively associated with malignant aggressiveness via upregulation of cell proliferation, migration, and vascular endothelial growth factors and cyclooxygenase-2 in prostate cancer. Transl Res 175: 116-128, 2016.

8. Pan M, Schinke H, Luxenburger E, Kranz G, Shakhtour J, Libl D, Huang Y, Gaber A, Pavšič M, Lenarčič B, et al: EpCAM ectodomain EpEX is a ligand of EGFR that counteracts EGF-mediated epithelial-mesenchymal transition through modulation of phospho-ERK1/2 in head and neck cancers. PLoS Biol 16: e2006624, 2018.

9. Yuan W, Liu B, Wang X, Li T, Xue H, Mo X, Yang S, Ding S and Han W: CMTM3 decreases EGFR expression and EGF-mediated tumorigenicity by promoting Rab5 activity in gastric cancer. Cancer Lett 386: 77-86, 2017.

10. Tsai PC, Fu YS, Chang LS and Lin SR: Taiwan cobra cardiotoxin III suppresses EGF/EGFR-mediated epithelial-to-mesenchymal transition and invasion of human breast cancer MDA-MB-231 cells. Toxicon 111: 108-120, 2016.

11. Clapéron A, Mergey M, Nguyen Ho-Bouldoires TH, Vignjevic D, Wendum D, Chrétien Y, Merabtene F, Frazao A, Paradis V, Housset C, et al: EGF/EGFR axis contributes to the progression of cholangiocarcinoma through the induction of an epithelial-mesenchymal transition. J Hepatol 61: 325-332, 2014.

12. Wang Y, Hu J, Wang Y, Ye W, Zhang X, Ju H, Xu D, Liu L, Ye D, Zhang L, et al: EGFR activation induced Snail-dependent EMT and myc-dependent PD-L1 in human salivary adenoid cystic carcinoma cells. Cell Cycle 17: 1457-1470, 2018. 
13. Jathal MK, Steele TM, Siddiqui S, Mooso BA, D'Abronzo LS, Drake CM, Whang YE and Ghosh PM: Dacomitinib, but not lapatinib, suppressed progression in castration-resistant prostate cancer models by preventing HER2 increase. Br J Cancer 121: 237-248, 2019.

14. Bender R and Gabhann FM: Dysregulation of the vascular endothelial growth factor and semaphorin ligand-receptor families in prostate cancer metastasis. BMC Syst Biol 9: 55, 2015.

15. Han W, Ding P, Xu M, Wang L, Rui M, Shi S, Liu Y, Zheng Y, Chen Y, Yang T and Ma D: Identification of eight genes encoding chemokine-like factor superfamily members 1-8 (CKLFSF1-8) by in silico cloning and experimental validation. Genomics 81 : 609-617, 2003.

16. Li H, Guo X, Shao L, Plate M, Mo X, Wang Y and Han W: CMTM5-v1, a four-transmembrane protein, presents a secreted form released via a vesicle-mediated secretory pathway. J Biochem Mol Biol 43: 182-187, 2010.

17. Liu B, Su Y, Li T, Yuan W, Mo X, Li H, He Q, Ma D and Han W: CMTM7 knockdown increases tumorigenicity of human non-small cell lung cancer cells and EGFR-AKT signaling by reducing Rab5 activation. Oncotarget 6: 41092-41107, 2015.

18. Li H, Li J, Su Y, Fan Y, Guo X, Li L, Su X, Rong R, Ying J and Mo X, et al: A novel 3p22.3 gene CMTM7 represses oncogenic EGFR signaling and inhibits cancer cell growth. Oncogene 33: 3109-3118, 2014

19. Both J, Krijgsman O, Bras J, Schaap GR, Baas F, Ylstra B and Hulsebos TJ: Focal chromosomal copy number aberrations identify CMTM8 and GPR177 as new candidate driver genes in osteosarcoma. PLoS One 9: e115835, 2014.

20. Chang SS and Amin MB: Utilizing the tumor-node-metastasis staging for prostate cancer: The sixth edition, 2002. CA Cancer J Clin 58: 54-59, 2008.

21. Livak KJ and Schmittgen TD: Analysis of relative gene expression data using real-time quantitative PCR and the 2(-Delta Delta C(T)) method. Methods 25: 402-408, 2001.

22. Shao L, Cui Y, Li H, Liu Y, Zhao H, Wang Y, Zhang Y, Ng KM, Han W, Ma D and Tao Q: CMTM5 exhibits tumor suppressor activities and is frequently silenced by methylation in carcinoma cell lines. Clin Cancer Res 13: 5756-5762, 2007.

23. Guo X, Li T, Wang Y, Shao L, Zhang Y, Ma D and Han W: CMTM5 induces apoptosis of pancreatic cancer cells and has synergistic effects with TNF-alpha. Biochem Biophys Res Commun 387: 139-142, 2009.
24. Shao L, Guo X, Plate M, Li T, Wang Y, Ma D and Han W: CMTM5-v1 induces apoptosis in cervical carcinoma cells. Biochem Biophys Res Commun 379: 866-871, 2009.

25. Huang ZM, Li PL, Yang P, Hou XD, Yang YL, Xu X and Xu F: Overexpression of CMTM7 inhibits cell growth and migration in liver cancer. Kaohsiung J Med Sci 35: 332-340, 2019.

26. Montanari M, Rossetti S, Cavaliere C, D'Aniello C, Malzone MG, Vanacore D, Di Franco R, La Mantia E, Iovane G, Piscitelli R, et al: Epithelial-mesenchymal transition in prostate cancer: An overview. Oncotarget 8: 35376-3589, 2017.

27. Wang L, Song G, Tan W, Qi M, Zhang L, Chan J, Yu J, Han J and Han B: MiR-573 inhibits prostate cancer metastasis by regulating epithelial-mesenchymal transition. Oncotarget 6: 35978-35990, 2015.

28. Lai Y, Kong Z, Zeng T, Xu S, Duan X, Li S, Cai C, Zhao Z and Wu W: PARP1-siRNA suppresses human prostate cancer cell growth and progression. Oncol Rep 39: 1901-1909, 2018.

29. Nieto MA, Huang RY, Jackson RA and Thiery JP: EMT: 2016. Cell 166: 21-45, 2016.

30. Hu F, Yuan W, Wang X, Sheng Z, Yuan Y, Qin C, He C and $\mathrm{Xu}$ T: CMTM3 is reduced in prostate cancer and inhibits migration, invasion and growth of LNCaP cells. Clin Transl Oncol 17: 632-639, 2015.

31. Guan L, Ji D, Liang N, Li S and Sun B: Up-regulation of miR-10b-3p promotes the progression of hepatocellular carcinoma cells via targeting CMTM5. J Cell Mol Med 22: 3434-3441, 2018.

32. Cai B, Xiao Y, Li Y and Zheng S: CMTM5 inhibits renal cancer cell growth through inducing cell-cycle arrest and apoptosis. Oncol Lett 14: 1536-1542, 2017.

33. Chen H, Zhou L, Wu X, Li R, Wen J, Sha J and Wen X: The PI3K/AKT pathway in the pathogenesis of prostate cancer. Front Biosci (Landmark Ed) 21: 1084-1091, 2016.

34. Xiao Y, Yuan Y, Zhang Y, Li J, Liu Z, Zhang X, Sheng Z, Xu T and Wang X: CMTM5 is reduced in prostate cancer and inhibits cancer cell growth in vitro and in vivo. Clin Transl Oncol 17: 431-437, 2015.

35. Yu JS and CUI W: Proliferation, survival and metabolism: The role of PI3K/AKT/mTOR signalling in pluripotency and cell fate determination. Development 143: 3050-3060, 2016.

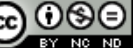

This work is licensed under a Creative Commons Attribution-NonCommercial-NoDerivatives 4.0 International (CC BY-NC-ND 4.0) License. 